\title{
Stabilization Mechanism for a Nonfibrillar Amyloid $\beta$ Oligomer Based on Formation of a Hydrophobic Core Determined by Dissipative Particle Dynamics
}

Ryoko Kawai ${ }^{1}$, Shuntaro Chiba ${ }^{2}$, Koji Okuwaki ${ }^{3}$, Ryo Kanada ${ }^{4}$, Hideo Doi ${ }^{5}$, Masahiro Ono ${ }^{6}$, Yuji Mochizuki $^{3,7}$, Yasushi Okuno*1,2,4

${ }^{1}$ Graduate School of Medicine, Kyoto University, 53 Shogoin-Kawahara-cho, Sakyo-ku, Kyoto 606-8507, Japan

2 RIKEN Medical Sciences Innovation Hub Program, 1-7-22 Suehiro-cho, Tsurumi-ku, Yokohama 230-0045, Japan

${ }^{3}$ Department of Chemistry and Research Center for Smart Molecules, Faculty of Science, Rikkyo University, 3-34-1 Nishi-ikebukuro, Toshima-ku, Tokyo 171-8501, Japan

4 Compass to Healthy Life Research Complex Program, RIKEN, 6-7-1 Minatojima Minami-machi, Chuo-ku, Kobe, Hyogo 650-0047, Japan

${ }^{5}$ National Institute of Advanced Industrial Science and Technology (AIST), 1-1-1 Umezono, Tsukuba, Ibaraki 305-8568, Japan

${ }^{6}$ Graduate School of Pharmaceutical Sciences, Kyoto University, 46-29 Shimodachi-cho, Sakyo-ku, Kyoto 606-8501, Japan

${ }^{7}$ Institute of Industrial Science, The University of Tokyo, 4-6-1 Komaba, Meguro-ku, Tokyo 153-8505, Japan 


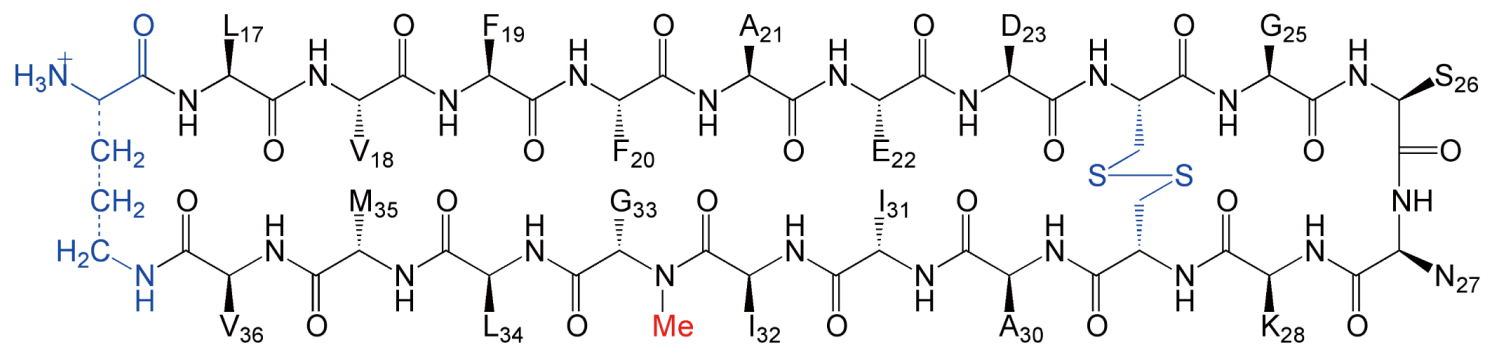

Figure S1. Chemical structure of the $A \beta_{17-36} \beta$-hairpin peptide in the crystal structure. ${ }^{l}$ Residues at positions 24 and 29 are mutated to cysteine and connect by a disulfide bond (shown in blue).

Two $\beta$-strands of $A \beta_{17-24}$ and $A \beta_{30-37}$ are covalently bound with $\delta$ Orn (shown in blue). Gly33 is $\mathrm{N}$-methylated (shown in red). 


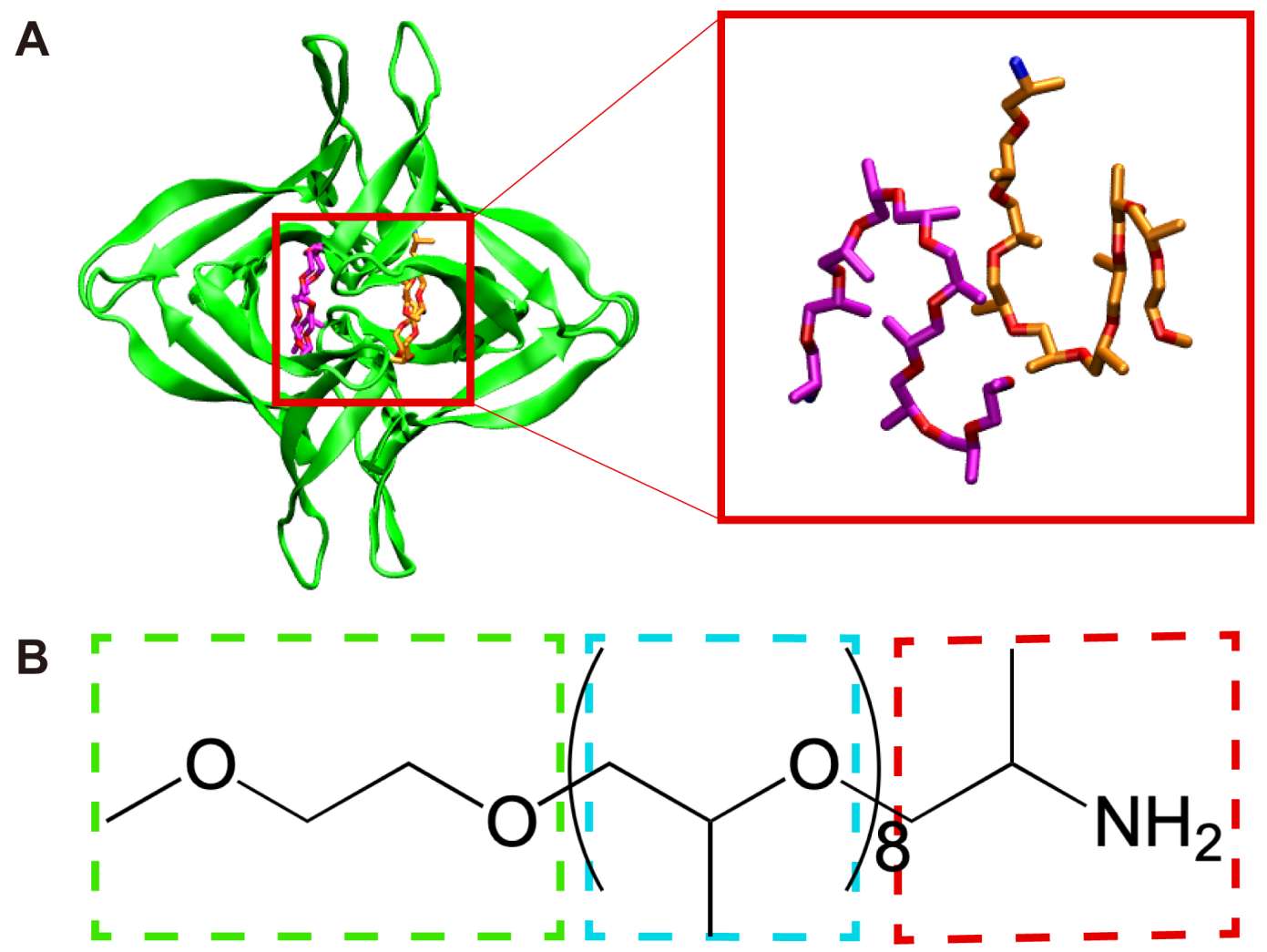

Figure S2. (A) Two molecules of Jeffamine-M600 in the central cavity of the $A \beta_{17-36}$ dodecamer in the crystal structure. Colors and representations of the dodecamer and Jeffamine M-600 follow that of Figure 1A. (B) Chemical structure of Jeffamine M-600. The 2-methoxyethoxy, 2-aminopropyl, and propylene glycol units are surrounded by dashed lines in green, red, and cyan, respectively, and are expressed as individual $\mathrm{S}$ beads, for a total of $10 \mathrm{~S}$ beads per Jeffamine-M600 molecule. 


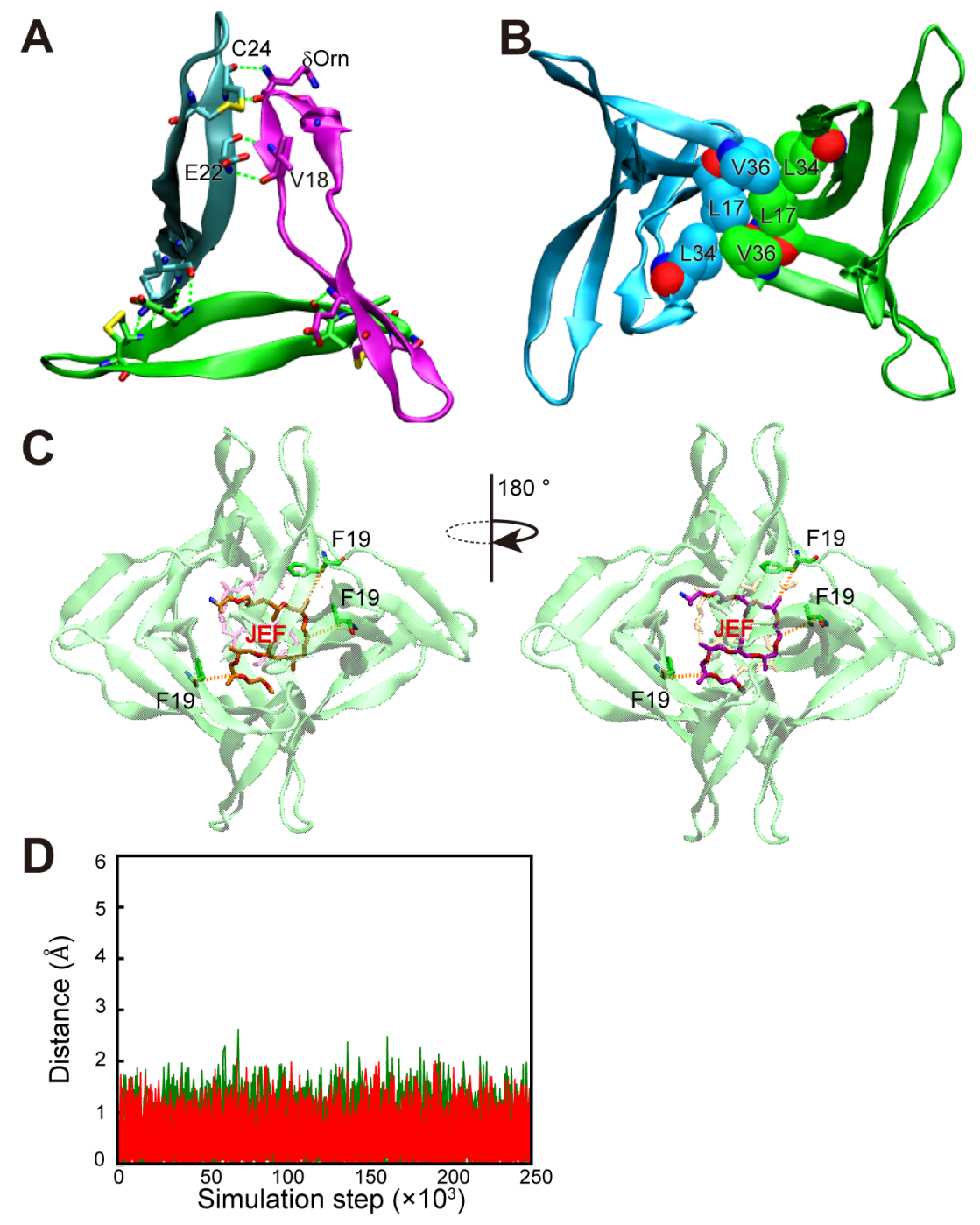

Figure S3. (A) Intermolecular hydrogen bonds (green dashed line) between the main chains of $\delta$ Orn and Cys 24 and Val18 and Glu22 at the corner of a triangular trimer unit found in the $A \beta_{17-}$ 36 dodecamer of the crystal structure. Residues $\delta$ Orn, Cys24, Val18, and Glu22 are shown by sticks (magenta/cyan/green, carbon; red, oxygen; blue, nitrogen; yellow, sulfur). (B) Hydrophobic packing between the side chains of Leu17, Leu34, and Val36 between the trimer units. These residues are represented by the CPK model (cyan/green, carbon; red, oxygen; blue, nitrogen). (C) Three Phe19 residues in the dodecamer contact closely to one Jeffamine M-600. The Phe19 residues neighboring Jeffamine M-600 are represented by stick models (green, carbon; red, oxygen; blue, nitrogen). (D) The vertical axis represents $\left|r_{i j}-r_{\mathrm{e}}\right|$, where $r_{i j}$ is the distances under restraints imposed on interactions shown in Figures $2 \mathrm{~A}$ and 2B, i.e., two 
pairs of residues at the three corners of the triangular unit and three pairs of residues between triangular units at six vertices, which are shown by red and green lines, respectively, and $r_{\mathrm{e}}$ is the equilibrium distances. 


\section{All-atom molecular dynamics simulation}

To assess the ability of DPD simulations to reproduce biomolecular structures compared with conventional all-atom molecular dynamics (MD) simulation, we conducted an all-atom MD simulation of the crystal structure of the dodecameric $A \beta_{17-36}$ peptide (Figure 1A). We constructed an initial structure of the all-atom MD simulation from the crystal structure using the Structure Preparation module of MOE 2018.1. Force field parameters for amino acids and Jeffamine M-600 were assigned from $\mathrm{ff} 14 \mathrm{SB}^{2}$ and from a parameterization method that combines the extended Hückel theory ${ }^{3}$ and the quantitative structure-property relationship model. The resulting parameter and coordinate files were converted to GROMACS-compatible files using the acpype program. ${ }^{4}$ The dodecameric structure was solvated in a TIP3P water ${ }^{5}$ box having $10 \AA$ larger sizes for the $x, y$, and $z$ directions of the molecule. Some water molecules were substituted by sodium or chloride ions to give a $100 \mathrm{mM}$ salt concentration for a neutralized system. The final box consisted of 1 dodecamer, 2 Jeffamine M-600, 13,302 waters, 27 sodium ions, and 29 chloride ions.

The system was subject to energy minimization by the steepest descent algorithm, followed by sequential 100-ps equilibrium simulations in the NVT and NPT ensembles with position restraints on heavy atoms in the dodecamer and Jeffamine M-600, and a harmonic potential with a force constant of $10 \mathrm{~kJ} \mathrm{~mol}^{-1} \AA^{-2}$. The temperature and pressure were controlled at $300 \mathrm{~K}$ by the velocity rescaling algorithm ${ }^{6}$ and at 1 bar by Parrinello-Rahman pressure coupling. ${ }^{7}$ After equilibrium, a 335 -ns simulation in the NPT ensemble was conducted, and the results were used for the RMSD analysis.

We used GROMACS $2019.1^{8}$ for minimization and MD simulation, in which the particle mesh Ewald method ${ }^{9,10}$ was applied to the electrostatic potential and a $10 \AA$ cutoff was applied to the vdW potential. The geometry of water molecules was fixed using the SETTLE algorithm. ${ }^{I I}$ All other bond lengths involving hydrogen atoms were fixed using the P-LINCS algorithm. ${ }^{12,13}$ 


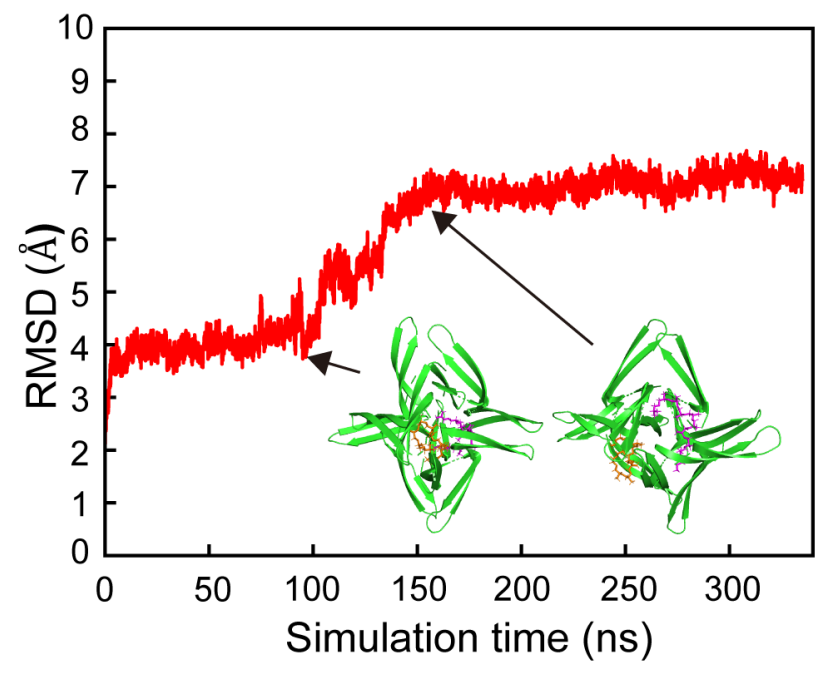

Figure S4. Result of all-atom MD simulation starting from the crystal structure. The overall Ca root-mean-square deviation (RMSD) with respect to the initial structure of the $A \beta_{17-36}$ dodecamer was approximately $4 \AA$ during the 100 -ns MD simulation. During 100-150 ns, the octahedron structure slightly collapsed, probably because one Jeffamine M-600 molecule spooled out from the center of the dodecamer. Imposing positional restraints relative to the center of the dodecamer would contribute to stabilizing the overall structure. As we did not impose positional restraints during the MD simulation, we regarded the simulation length during $0-100 \mathrm{~ns}$ as equilibrated sampling of the dodecamer with the octahedron structure. 

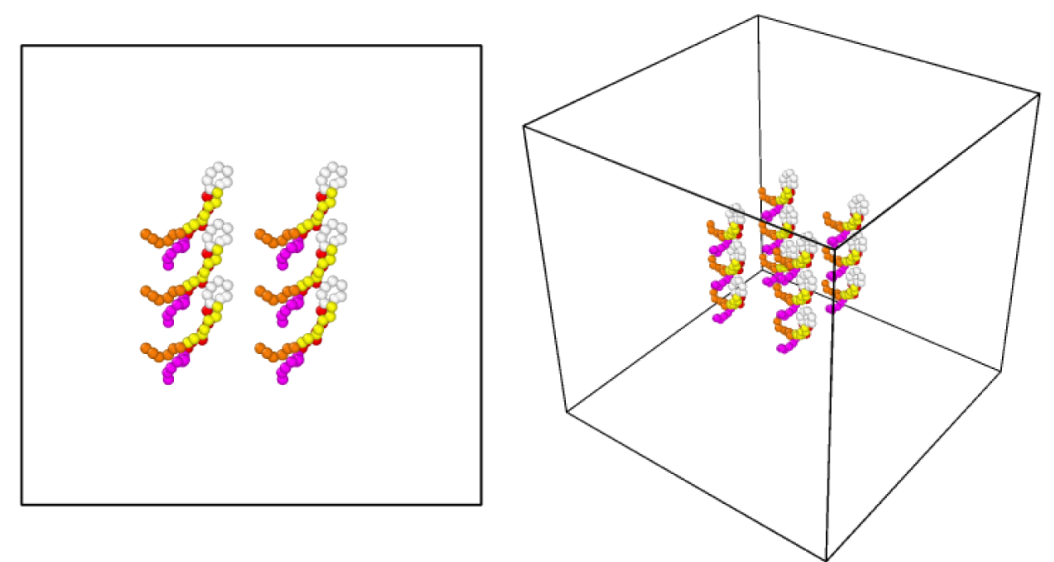

Figure S5. The initial structure for $A \beta_{9-42}$ DPD simulation without showing $\mathrm{W}$ beads. Twelve $\mathrm{A} \beta_{9-42}$ peptides were placed separately. Color schemes for DPD beads follow Figure 4 . 

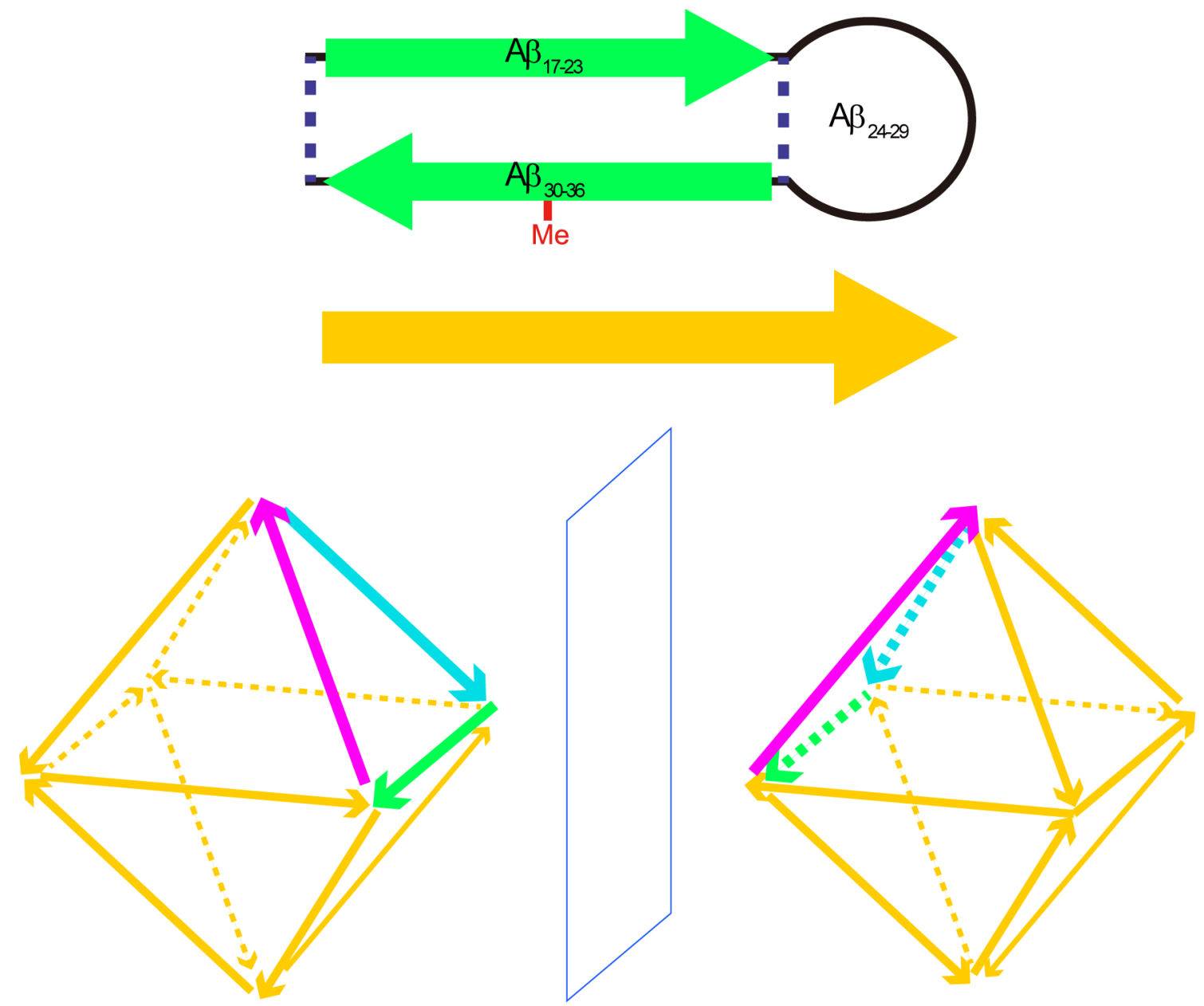

Figure S6. The distance restraints can be satisfied in the real (bottom left) or mirror image (bottom right) of the crystal structure. The direction of the orange arrow is defined as that of the $\mathrm{A} \beta_{17-23} \beta$-strand (top). Both structures were produced by DPD simulation using the initial coordinates in which $12 \mathrm{~A} \beta$ peptides are placed separately (Figures 4B and S5). 


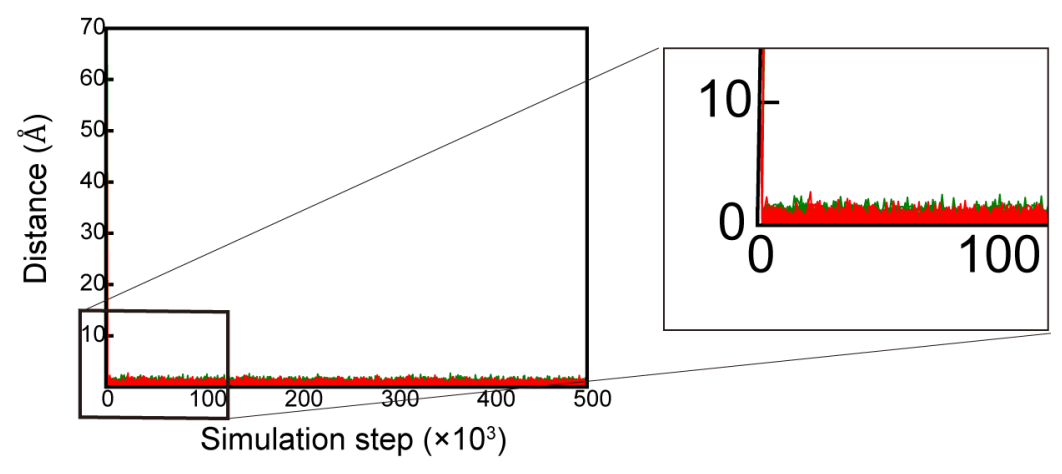

Figure S7. Distances under restraints converged to equilibrium distances (see Figure S3D). Plots for distance restraints at the corner of a triangular trimer unit (Figure 2A) and between trimer units (Figure 2B) are shown by red and green lines, respectively. 


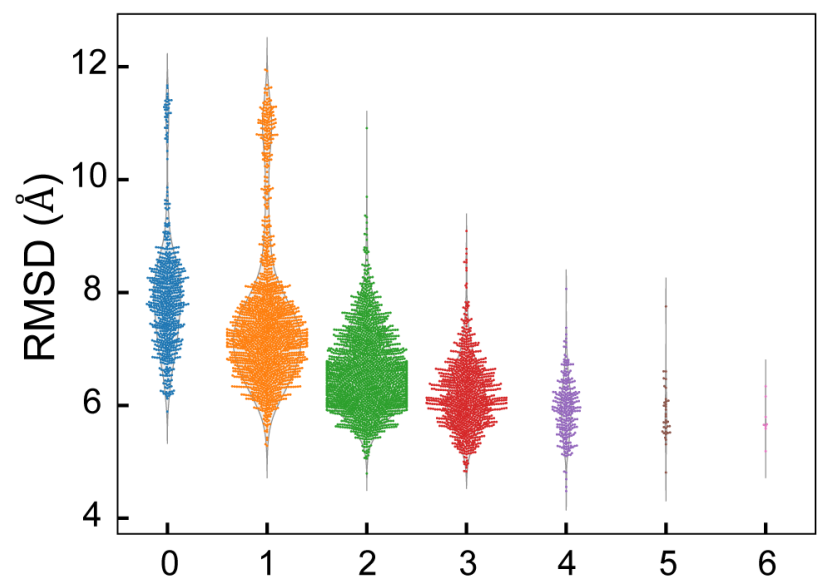

Number of C-terminal regions in central cavity

Figure S8. Violin plot for assessing the relation between the number of C-terminal regions in the core and RMSD, drawn by collecting snapshots over the last $400 \times 10^{3}$ steps for independent simulations, excluding one simulation whose average RMSD value was an outlier. 


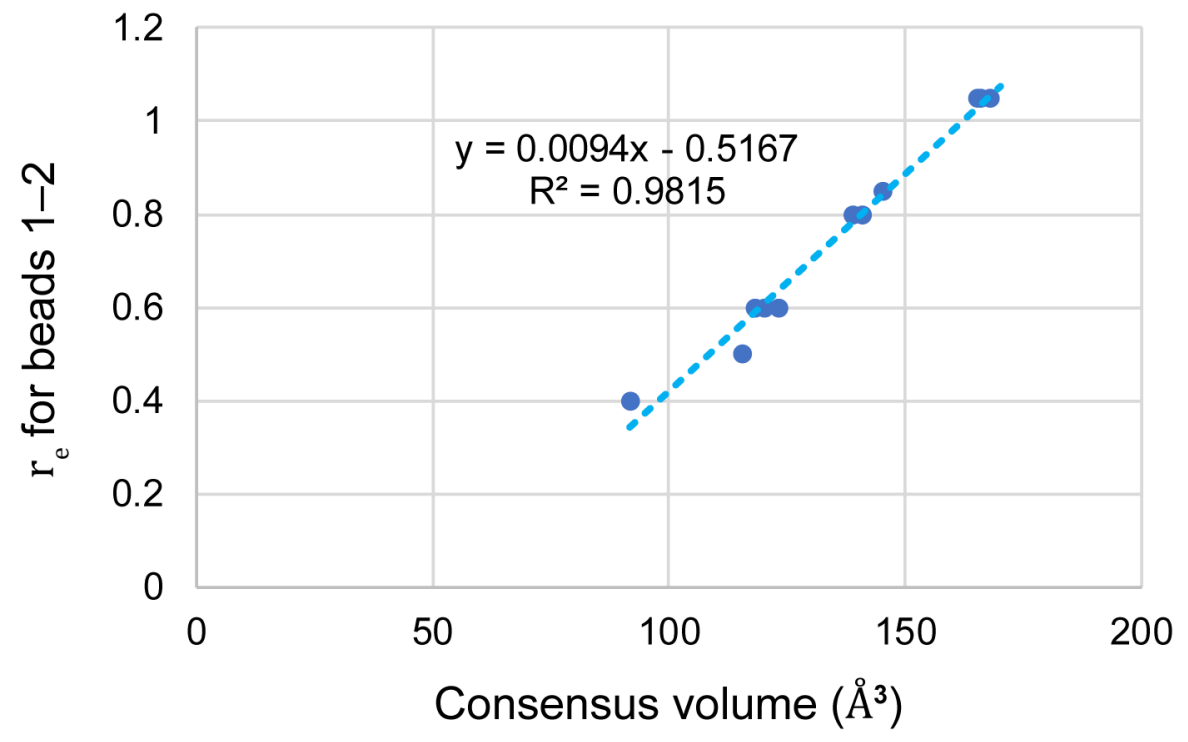

Figure S9. Correlation between the equilibrium distances of side-chains and the effective amino acid volumes. Equilibrium distances $r_{\mathrm{e}}$ for beads $1-2$ not provided in the reference ${ }^{14}$ were determined from this regression line between $r_{\mathrm{e}}$ for beads 1-2 and the amino acid consensus volumes. See the definition for the consensus volume in the literature. ${ }^{15}$ 
Table S1. Average RMSD values calculated from 15 independent simulations of wild type, G37C, G38C, V39C, V40C, I41C, and A42C.

\begin{tabular}{|c|c|c|c|c|c|c|c|}
\hline Simulation ID & Wild & G37C & G38C & V39C & V40C & $\mathrm{I} 41 \mathrm{C}$ & $\mathrm{A} 42 \mathrm{C}$ \\
\hline 1 & 6.50 & 6.35 & 6.52 & 7.47 & 7.70 & 6.88 & 7.29 \\
\hline 2 & 6.37 & 6.49 & 6.55 & 6.51 & 6.55 & 6.33 & 10.60 \\
\hline 3 & 12.42 & 7.27 & 6.22 & 7.98 & 6.33 & 7.59 & 6.60 \\
\hline 4 & 6.99 & 6.30 & 7.03 & 9.67 & 7.81 & 6.76 & 6.55 \\
\hline 5 & 6.10 & 6.36 & 9.80 & 7.55 & 7.83 & 7.00 & 7.81 \\
\hline 6 & 6.36 & 9.24 & 6.58 & 6.33 & 7.22 & 7.17 & 10.22 \\
\hline 7 & 6.80 & 6.40 & 6.27 & 6.69 & 10.44 & 11.56 & 7.17 \\
\hline 8 & 8.02 & 6.10 & 6.58 & 6.61 & 6.57 & 10.27 & 7.51 \\
\hline 9 & 6.22 & 6.81 & 6.47 & 7.13 & 7.19 & 6.06 & 7.33 \\
\hline 10 & 6.24 & 6.29 & 6.26 & 6.91 & 12.58 & 9.17 & 7.71 \\
\hline 11 & 6.37 & 6.62 & 6.38 & 6.46 & 7.38 & 6.55 & 7.39 \\
\hline 12 & 7.59 & 6.59 & 6.41 & 10.37 & 11.62 & 7.15 & 6.64 \\
\hline 13 & 5.92 & 6.47 & 6.35 & 6.46 & 6.69 & 8.05 & 9.32 \\
\hline 14 & 6.36 & 6.51 & 6.58 & 7.08 & 6.71 & 6.82 & 7.24 \\
\hline 15 & 6.03 & 7.08 & 7.32 & 7.35 & 6.65 & 7.20 & 10.67 \\
\hline Mean & $\begin{array}{c}6.95 \\
(6.56)\end{array}$ & $\begin{array}{c}6.73 \\
(6.55)\end{array}$ & $\begin{array}{c}6.75 \\
(6.54)\end{array}$ & 7.37 & 7.95 & 7.64 & 8.00 \\
\hline SD & $\begin{array}{c}1.62 \\
(0.58) \\
\end{array}$ & $\begin{array}{c}0.76 \\
(0.31) \\
\end{array}$ & $\begin{array}{c}0.89 \\
(0.29)\end{array}$ & 1.18 & 1.96 & 1.54 & 1.45 \\
\hline
\end{tabular}

The average RMSD of each simulation was calculated over the last $200 \times 10^{3}$ steps. One simulation each for wild type, G37C, or G38C, whose average RMSD value was detected as an outlier, is shown in red italics, i.e., RMSD $>$ Mean $+3 \times$ SD. Mean and SD calculated without the outlier are given in parenthesis. 
Table S2. Parameters for 1-2 harmonic bonds

\begin{tabular}{cccccc}
\hline & Bead 1 & Bead 2 & Bead 3 & $\begin{array}{c}r_{\mathrm{e}} \text { for } \\
\text { beads 1-2 }\end{array}$ & $\begin{array}{c}r_{\mathrm{e}} \text { for } \\
\text { beads 2-3 }\end{array}$ \\
\hline Ala & $\mathrm{T}$ & & & & \\
Asn & $\mathrm{S}$ & $\mathrm{H}$ & & 0.6 & \\
Asp & $\mathrm{S}$ & $\mathrm{H}$ & & 0.5 & \\
Cys(SS) & $\mathrm{S}$ & $\mathrm{T}$ & & 0.5 & \\
Cys(SH) & $\mathrm{S}$ & $\mathrm{H}$ & & 0.5 & \\
Gln & $\mathrm{S}$ & $\mathrm{H}$ & & 0.85 & \\
Glu & $\mathrm{S}$ & $\mathrm{H}$ & & 0.8 & \\
Gly & $\mathrm{S}$ & & & & \\
His & $\mathrm{S}$ & $\mathrm{H}$ & & 0.95 & \\
Ile & $\mathrm{S}$ & $\mathrm{T}$ & & 1.05 & \\
Leu & $\mathrm{S}$ & $\mathrm{T}$ & & 1.05 & \\
Lys & $\mathrm{S}$ & $\mathrm{T}$ & $\mathrm{H}$ & 0.5 & 0.7 \\
Met & $\mathrm{S}$ & $\mathrm{T}$ & & 1.05 & \\
Phe & $\mathrm{S}$ & $\mathrm{T}$ & $\mathrm{T}$ & 0.6 & 0.6 \\
Ser & $\mathrm{S}$ & $\mathrm{H}$ & & 0.4 & \\
Tyr & $\mathrm{S}$ & $\mathrm{T}$ & $\mathrm{H}$ & 0.8 & 0.6 \\
Val & $\mathrm{S}$ & $\mathrm{T}$ & & 0.8 & \\
Orn & $\mathrm{S}$ & $\mathrm{T}$ & $\mathrm{H}$ & 0.6 & 0.6 \\
\hline
\end{tabular}

The force constant $\mathrm{C}$ was set to 160 . For the equilibrium distance between $\mathrm{S}$ beads of the peptide main chain and Jeffamine M-600, $r_{\mathrm{e}}=0.6$ was used. Equilibrium distances between beads of the main chain and side-chains, $r_{\mathrm{e}}$ for beads $1-2$ and 2-3 were derived using Vishnyakov et al. ${ }^{14}$ Because the study did not provide $r_{\mathrm{e}}$ for $1-2$ of Cys and His, we derived these values based on the correlation between $r_{\mathrm{e}}$ for beads $1-2$ and the effective amino acid volumes ${ }^{15}$ (Figure S9). 


\section{References}

[1] Kreutzer, A. G., Hamza, I. L., Spencer, R. K., and Nowick, J. S. (2016) X-ray Crystallographic Structures of a Trimer, Dodecamer, and Annular Pore Formed by an A $\beta_{17-36} \beta$-Hairpin, J. Am. Chem. Soc. 138, 4634-4642.

[2] Maier, J. A., Martinez, C., Kasavajhala, K., Wickstrom, L., Hauser, K. E., and Simmerling, C. (2015) ff14SB: Improving the Accuracy of Protein Side Chain and Backbone Parameters from ff99SB, J. Chem. Theory Comput. 11, 3696-3713.

[3] Gerber, P. R., and Muller, K. (1995) MAB, a generally applicable molecular force field for structure modelling in medicinal chemistry, J. Comput. Aided Mol. Des. 9, 251-268.

[4] Sousa da Silva, A. W., and Vranken, W. F. (2012) ACPYPE - AnteChamber PYthon Parser interfacE, BMC Res. Notes 5, 367.

[5] Jorgensen, W. L., Chandrasekhar, J., Madura, J. D., Impey, R. W., and Klein, M. L. (1983) Comparison of simple potential functions for simulating liquid water, J. Chem. Phys. 79, 926-935.

[6] Bussi, G., Donadio, D., and Parrinello, M. (2007) Canonical sampling through velocity rescaling, J. Chem. Phys. 126, 014101.

[7] Parrinello, M., and Rahman, A. (1981) Polymorphic transitions in single crystals: A new molecular dynamics method, J. Appl. Phys. 52, 7182-7190.

[8] Abraham, M. J., Murtola, T., Schulz, R., Páll, S., Smith, J. C., Hess, B., and Lindahl, E. (2015) GROMACS: High performance molecular simulations through multi-level parallelism from laptops to supercomputers, SoftwareX 1-2, 19-25.

[9] Essmann, U., Perera, L., Berkowitz, M. L., Darden, T., Lee, H., and Pedersen, L. G. (1995) A smooth particle mesh Ewald method, J. Chem. Phys. 103, 8577-8593.

[10] Darden, T., York, D., and Pedersen, L. (1993) Particle mesh Ewald: An N·log(N) method for Ewald sums in large systems, J. Chem. Phys. 98, 10089-10092. 
[11] Miyamoto, S., Shuichi, and Kollman, P. A. (1992) Settle: An analytical version of the SHAKE and RATTLE algorithm for rigid water models, J. Comput. Chem. 13, 952962.

[12] Hess, B., Bekker, H., Berendsen, H. J. C., and Fraaije, J. G. E. M. (1997) LINCS: A linear constraint solver for molecular simulations, J. Comput. Chem. 18, 1463-1472.

[13] Hess, B. (2008) P-LINCS: A Parallel Linear Constraint Solver for Molecular Simulation, J. Chem. Theory Comput. 4, 116-122.

[14] Vishnyakov, A., Talaga, D. S., and Neimark, A. V. (2012) DPD Simulation of Protein Conformations: From $\alpha$-Helices to $\beta$-Structures, J. Phys. Chem. Lett. 3, 3081-3087.

[15] Perkins, S. J. (1986) Protein volumes and hydration effects. The calculations of partial specific volumes, neutron scattering matchpoints and 280-nm absorption coefficients for proteins and glycoproteins from amino acid sequences, Eur. J. Biochem. 157, 169180. 